\title{
Previsão climática de precipitação para a região Sul por rede neural autoconfigurada
}

\author{
Climate precipitation prediction for South region by neural network \\ self-configured
}

\author{
Juliana Aparecida Anochi e Haroldo Fraga de Campos Velho \\ Instituto Nacional de Pesquisas Espaciais - INPE \\ juliana.anochi@gmail.com; haroldo@lac.inpe.br
}

\begin{abstract}
Resumo
Previsão climática do campo de precipitação é um aspecto chave, pois esta é uma variável meteorológica difícil, de grande variabilidade temporal e espacial, com forte impacto para a sociedade. Um método baseado em rede neural artificial é aplicado para previsão mensal e sazonal de precipitação na região Sul do Brasil. O uso de redes neurais como um modelo preditivo é bastante difundido em diferentes aplicações. A melhor configuração para a rede neural é calculada automaticamente. O esquema de autoconfiguração é descrito como um problema de otimização.
\end{abstract}

Palavras-chave: Previsão climática, precipitação, rede neural, otimização, redução de dados.

\begin{abstract}
Climate prediction for precipitation field is a key issue, because such meteorological variable is the challenge for climate and weather forecasting due to the high spatial and temporal variability with strong impact on the society. A method based on the artificial neural network is applied to monthly and seasonal precipitation forecast in southern Brazil. The use of neural networks as a predictive model is widespread in different applications. The best configuration for the neural network is automatically calculated. The autoconfiguration scheme is described as an optimization problem.
\end{abstract}

Keywords: Climate prediction, precipitation, neural network, optimazation, data reduction. 


\section{Introdução}

A previsão climática do campo de precipitação é um fator essencial para os setores que são afetados pela variabilidade climática, como a produção de energia, atividades agrícolas, geração de energia hidroelétrica, transportes e recentemente à defesa civil. Tendo em vista que o Brasil é um país com grande extensão territorial, com diferenciados regimes de precipitação e temperatura, a implementação de modelos para o monitoramento climático já é pauta em pesquisas relacionadas ao tema por Anochi (2010); Sousa e Sousa (2010); Baboo e Shereef (2010).

O clima se diferencia de Norte a Sul no Brasil. Na região Norte, verifica-se um clima equatorial chuvoso, praticamente sem estação seca. No Nordeste, a estação chuvosa, com baixos índices pluviométricos, restringe-se a poucos meses, caracterizando um clima semi-árido e apresenta alta previsibilidade climática. As regiões Sudeste e Centro-Oeste sofrem influência tanto de sistemas tropicais como de latitudes médias, com estação seca bem definida no inverno e estação chuvosa no verão com chuvas convectivas. Ambas regiões apresentam baixa previsibilidade devido à menor dependência com relação as condições dos oceanos e à grande variedade dos sistemas meteorológicos que as atingem. Finalmente, a região Sul do Brasil é caracterizada com média previsibilidade, e devido à sua localização latitudinal, sofre mais influência dos sistemas de latitudes médias, onde os sistemas frontais são os principais causadores de chuvas durante o ano Sampaio e Silva Dias (2014).

Essa dependência dos fatores climáticos motivou o trabalho de Anochi e Silva (2009), em que desenvolveu um modelo de previsão climática do campo de precipitação por redes neurais. Esse modelo foi adaptado para o campo de precipitação para previsão mensal e sazonal, proporcionando a elaboração de cenários futuros que apoiam os estudos de vulnerabilidade e permite a elaboração de projeções dos extremos climáticos do estado atmosférico.

\section{Rede neural autoconfigurada}

Redes neurais (NN) são métodos computacionais cujo princípio de funcionamento é regido por um modelo matemático inspirado no funcionamento dos elementos básicos que formam a estrutura neural de organismos inteligentes, que adquirem conhecimento através de experiência. Seu comportamento resulta das interações entre as unidades de processamento, a partir de seu ambiente, através de um processo de aprendizagem. $\mathrm{O}$ uso de redes neurais, surgiram como excelentes ferramentas para derivar modelos de dados, devido à sua característica inerente de plasticidade que permite a adaptação da tarefa de aprendizagem quando os dados são fornecidos.

Computacionalmente, rede neural é um sistema paralelo distribuído, formado por neurônios, os quais podem ser distribuídos em uma ou mais camadas conectadas por conexões (pesos sinápticos), em que armazenam o conhecimento representado no modelo e servem para ponderar a entrada recebida por cada neurônio da rede Haykin (1998).

Existem vários tipos de NN que diferem em arquitetura ou forma de treinamento. Para o problema de previsão climática, proposto neste trabalho, utilizou-se a rede Perceptron de Múltiplas Camadas (MLP) com treinamento supervisionado usando algoritmo de retropropagação do erro (backpropagation).

\subsection{Configuração ótima por meta-heurística}

A definição de parâmetros da topologia da rede do tipo MLP, a qual é alvo deste trabalho, trata-se basicamente dos seguintes parâmetros: número de camadas escondidas (intermediária), número de neurônios em cada camada, tipo da função de ativação, taxa de aprendizagem e taxa momentum.

O processo empírico para encontrar uma boa arquitetura para uma NN é um procedimento padrão, conhecido também como tentativa e erro Prechelt (1994). Uma outra alternativa é formular o problema de identificação de uma topologia ótima como um problema de otimização. Assim, a formulação para uma rede autoconfigurável tem como finalidade determinar o melhor conjunto de parâmetros da NN minimizando uma função objetivo.

A solução do problema de otimização é endereçada pelo Algoritmo de Colisão de Múltiplas Partículas (MPCA) - ver Luz et al. (2008), para determinar os parâmetros ótimos. A função objetivo definida por Carvalho et al. (2011) é composta pela soma ponderada dos erros de treinamento e generalização multiplicado por uma penalidade, representada na Equação 1.

$$
J=\text { penalidade } \times \frac{\rho_{1} E_{\mathrm{train}}+\rho_{2} E_{\mathrm{gen}}}{\rho_{1}+\rho_{2}},
$$

em que $\rho_{1}=1$ e $\rho_{2}=0,1$, os mesmos valores proposto por Carvalho et al. (2011). Estes são termos que modificam a relevância atribuída aos erros de treinamento e generalização respectivamente. $\mathrm{O}$ valor mínimo de $J$ corresponde à arquitetura simples e com um bom desempenho.

O termo penalidade prioriza topologias menores evitando o overfitting, utilizando o número de neurônios presentes na camada intermediária ( $\left.n_{\text {neuronios }}\right)$ e o número total de épocas ( $\left.n_{\text {epocas}}\right)$ para alcançar a convergência. $\mathrm{O}$ overfitting ocorre quando a rede memoriza os padrões de treinamento, ao invés de aprender/extrair 
informações. A Equação 2 representa a penalidade:

$$
\text { penalidade }=C_{1} e^{\left(n_{\text {neuronios }}\right)^{2}}+C_{2}\left(n_{\text {epocas }}\right)+1 \text {, }
$$

em que $C_{1}=1$ e $C_{2}=0,1$, são parâmetros de ajuste para encontrar um equilíbrio nos fatores de medição da complexidade.

O algoritmo MPCA é empregado para otimizar cinco parâmetros de domínio misto de variáveis: dois contínuos (o parâmetro de taxa de aprendizagem $(\eta)$, e a constante momentum $(\alpha)$ ); e três discretos (número de camadas intermediárias ( $\left.n_{\text {camada }}\right)$, número de neurônios em cada camada intermediária ( $\left.n_{\text {neuronios }}\right)$ e o tipo de função de ativação $\left.\left(f_{\text {ativacao }}\right)\right)$. Os valores permitidos para estes parâmetros são apresentados na Tabela 1.

Tabela 1: Parâmetros que definem arquiteturas de NNs

\begin{tabular}{ccc}
\hline Tipo & Parâmetros & Valor \\
\hline Discreto & $n_{\text {camadas }}$ & {$[1,2]$} \\
& $n_{\text {neuronios }}$ & {$[1,40]$} \\
& $f_{\text {ativacao }}$ & $\begin{array}{c}\text { tangente (1) } \\
\text { sigmoide (2) } \\
\text { gaussiana (3) }\end{array}$ \\
\hline \multirow{2}{*}{ Contínuo } & $\eta$ & {$[0.01,1.0]$} \\
& $\alpha$ & {$[0.01,1.0]$} \\
\hline
\end{tabular}

\section{Experimentos}

\subsection{Dados Meteorológicos}

A área de estudo proposta abrange a região Sul (S) entre as longitudes $52^{\circ}, 38^{\circ} \mathrm{W}$ e latitudes $25^{\circ}, 15^{\circ} \mathrm{S}$, como ilustrado na Figura 1 (área em destaque no mapa do Brasil).

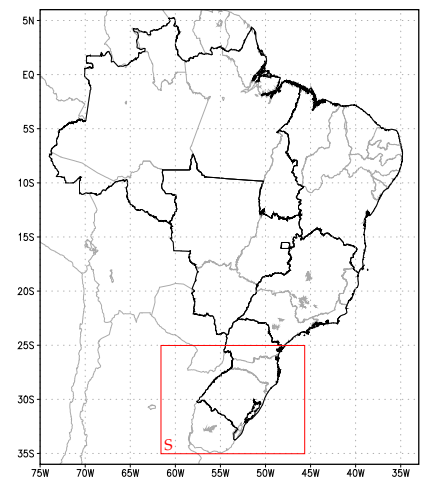

Figura 1: Área de estudo: Sul (S)

Os dados utilizados nos experimentos foram coletados da base de dados do NCEP/NCAR (National Centers for Environmental Prediction / The National Center for Atmospheric Research) ${ }^{1}$. Os dados coletados

\footnotetext{
${ }^{1}$ http://www.ncep.noaa.gov/
}

são mensais e abrangem o período de janeiro de 1990 a fevereiro de 2015 (302 meses). Trata-se de um conjunto de dados globais de reanálise com resolução espacial (latitude e longitude) de $2.5^{\circ} \times 2.5^{\circ}$.

A Tabela 2 traz uma breve descrição das variáveis meteorológicas selecionadas do NCEP/NCAR, com suas unidades e níveis (superfície ou níveis de pressão em $\mathrm{hPa}$ ), usadas neste estudo.

Tabela 2: Variáveis meteorológicas

\begin{tabular}{cc}
\hline Variável & Descrição \\
\hline $\mathbf{u}$ & Vento Zonal $-300,500,850 \mathrm{hPa}$ \\
$\mathbf{v}$ & Vento Meridional $-300,500,850 \mathrm{hPa}$ \\
temp & Temperatura $\left[{ }^{\circ} \mathrm{C}\right]$ \\
air & Temperatura na superfície $\left[{ }^{\circ} \mathrm{C}\right]$ \\
shum & Umidade Especifica $[\mathrm{g} / \mathrm{kg}]$ \\
prec & Precipitação \\
\hline
\end{tabular}

Durante a fase de treinamento da rede neural, a capacidade de generalização é avaliada através de um conjunto de dados de validação. Assim, os dados de treinamento são divididos em dois subconjuntos: exemplos e validação. O subconjunto de exemplos é usado no treinamento da rede. $\mathrm{O}$ treinamento é interrompido periodicamente após um número determinado de épocas, e a rede é testada com o subconjunto de validação (processo de validação cruzada). Na fase de generalização, um conjunto não utilizado na fase de treinamento é apresentado à rede para avaliação e verificação de seu desempenho. Desta perspectiva, o conjunto de dados foi dividido em três grupos:

- Conjunto de treinamento: janeiro de 1990 - dezembro de 2010;

- Conjunto de validação cruzada: janeiro - dezembro de 2011;

- Conjunto de generalização: janeiro de 2012 - fevereiro de 2015.

\subsection{Técnicas de redução}

Identificar, com razoável antecedência e confiabilidade, condições favoráveis à ocorrência de eventos climáticos e, realizar em tempo hábil para que se possa tomar as devidas medidas e consequentemente reduzir o impacto negativo de eventos meteorológicos, é algo essencial para a sociedade. Em meteorologia, dados de diversas fontes, tais como dados de satélites, dados observados (gerados por estações de medições em superfície, boias oceânicas, radiossondagens), modelos numéricos de previsão do tempo, dados de ocorrência de descargas elétricas, radar, dentre outros, são usados para realizações de previsões do tempo e clima. 
Entretanto, as previsões de eventos meteorológicos são complexas pela necessidade de análise de grande volume de dados. Desa forma, propõe-se o uso de uma técnica de mineração de dados a Teoria dos Conjuntos Aproximativos (TCA), para identificar as variáveis mais significativas para o processo de previsão climática.

Inicialmente a rede MLP foi treinada para realizar a previsão climática utilizando todas as variáveis disponíveis na base de dados. Posteriormente, os dados disponíveis para treinamento são pré-processados para extrair informações relevantes (em uma abordagem de mineração de dados).

A TCA foi proposta por Pawlak (1982), como um formalismo matemático, para o tratamento de informações incertas e imprecisas, por meio de uma relação de indiscernibilidade e avalia se os elementos de um conjunto são indiscerníveis, ou seja, se possuem as mesmas propriedades, em que considera que se tal relação existe entre dois ou mais objetos, significa que esses possuem os mesmos valores para todos os atributos que os caracterizam.

O processo de redução consiste em identificar os atributos que preservam a relação indiscernibilidade Komorowski et al. (1998); Øhrn e Komorowski (1997). Neste trabalho, a ferramenta Rosetta ${ }^{2}$ foi utilizada para realizar a redução de dimensionalidade dos dados meteorológicos.

\section{Resultados}

Os resultados levam em consideração a média de 15 experimentos, sendo que em cada experimento compreende 500 execuções diferentes do algoritmo. O critério de parada foi considerado o número máximo de avaliações da função objetivo, neste trabalho considerou-se 500 avaliações.

A Tabela 3 mostra a configuração ótima de parâmetros obtidos com o uso da meta-heurística MPCA.

Tabela 3: Parâmetros ótimos para topologia de NN

\begin{tabular}{ll}
\hline Parâmetros & Valor \\
\hline Camada intermediária & 1 \\
Número de neurônio & 15 \\
Taxa de aprendizagem $\eta$ & 0.53 \\
Taxa momentum $\alpha$ & 0.24 \\
Função de ativação & Logistica \\
\hline
\end{tabular}

As variáveis mais significativas obtidas pela TCA para a região Sul são apresentadas na Tabela 4. Verificase que do total de variáveis apresentadas na Tabela 2, sete foram extraídas para o processo de previsão de precipitação.

\footnotetext{
${ }^{2}$ http://ww.lcb.uu.se/tools/rosetta
}

Tabela 4: Variáveis extraídas pela TCA - Sul

\begin{tabular}{ll}
\hline Variável & $\%\left(f_{A}\right)$ \\
\hline temp & 77 \\
u300 & 79 \\
u850 & 80 \\
v300 & 77 \\
v500 & 79 \\
v850 & 71 \\
shum & 78 \\
\hline
\end{tabular}

\subsection{Previsão mensal}

A Figura 2 apresenta os resultados de previsão de precipitação mensal para o mês de Julho em 2014. A Figura 2(a) mostra o campo de precipitação observado no mês de Julho em 2014 extraído da base de dados do NCEP/NCAR. As Figuras 2(b) e 2(c) são os resultados de previsão de precipitação obtidos com diferentes NNs: os parâmetros da topologia foram configurados automaticamente pela meta-heurística MPCA e o treinamento levou em consideração todas as variáveis disponíveis na base de dados (NN-todas variáveis) e, a rede treinada com o conjunto reduzido de variáveis e configurada com os parâmetros ótimos, respectivamente.

As Figuras 2(d) e 2(e) apresentam os mapas de erro para as previsões de precipitação obtidos por: NN-todas variáveis e NN-TCA, respectivamente.

Analisando os resultados de previsão no mês de Julho, constata-se que ambos modelos de NNs atingiram padrões de precipitação semelhantes ao campo de observação. Entretanto, a rede 2(c) apresentou uma margem de erro inferior nos estados do Rio Grande do Sul e Santa Catarina - ver Figura 2(e).

\subsection{Previsão sazonal}

A Figura 3 apresenta os resultados de previsão de precipitação sazonal para a estação Inverno de 2014. A Figura 3(a) mostra o campo de precipitação observado para a estação Inverno de 2014 extraído da base de dados do NCEP/NCAR. As Figuras 3(b) e 3(c) são os resultados de previsão de precipitação obtidos com diferentes redes neurais: a topologia configurada automaticamente usando o MPCA (NN-todas variáveis) e usando todas as variáveis no treinamento e, a NN treinada com o conjunto reduzido de variáveis e configurada com os parâmetros ótimos (NN: TCA), respectivamente.

As Figuras 3(d) e 3(e) apresentam os mapas de erro para a previsão de precipitação usando os modelos: NNtodas variáveis e NN-TCA, respectivamente.

Tendo em vista os resultados obtidos, fica evidente que a melhor solução obtida foi pela NN-TCA, a qual obteve uma margem de erro inferior - ver Figura 3(e). Embora a rede 3(b)(NN-todas variáveis) siga um padrão 


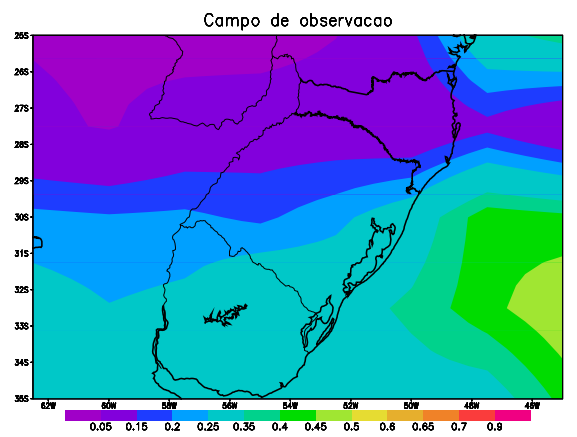

(a) Campo de observação: Julho

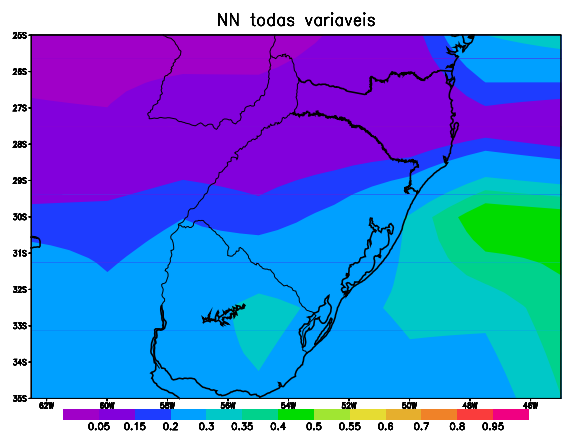

(b) Previsão: NN com todas variáveis

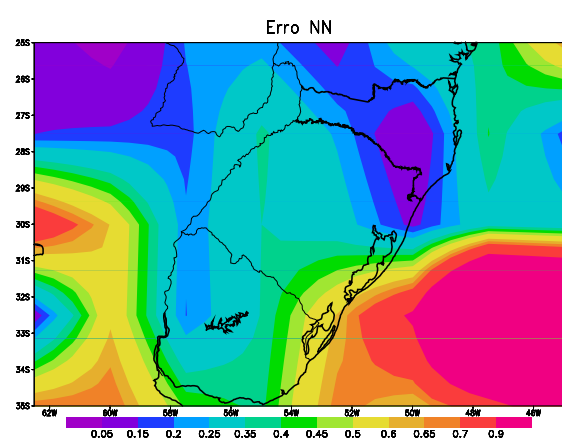

(d) Erro: NN

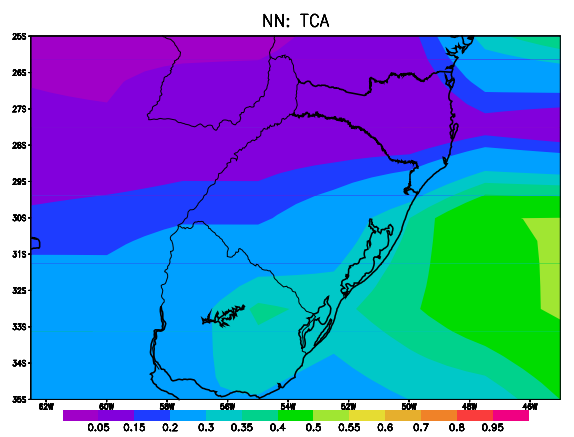

(c) Previsão: NN com redução TCA

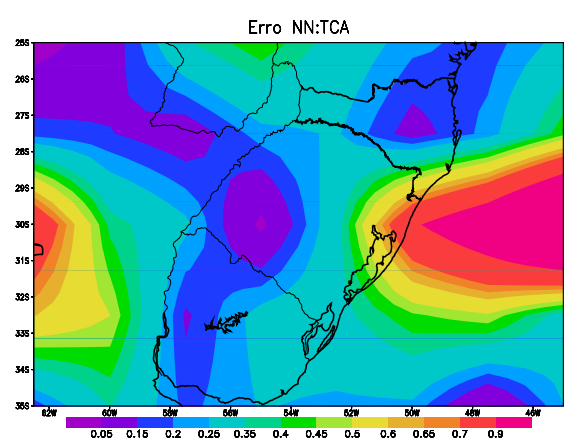

(e) erro: NN TCA

Figura 2: Previsão mensal de precipitação para o mês de Julho

de precipitação muito semelhante ao observado, possui um erro superior.

Salienta-se que houve uma melhora significativa na previsão com o uso da técnica TCA - ver Figura 3(c), a qual foi treinada com os parâmetros ótimos definidos por MPCA e com a redução de dimensionalidade (conjunto reduzido de variáveis).

\section{Conclusões}

Neste trabalho apresentamos um estudo que aborda o uso de uma meta-heurística na otimização de parâmetros de arquiteturas de redes neurais. O problema foi formulado como um processo de otimização, utilizando o algoritmo MPCA uma técnica estocástica empregada para tratar a solução do problema. Em geral, esses parâ- metros são determinados por experimentação empírica, em que é realizado diversas tentativas com diferentes topologias até atingir resultados satisfatórios, entretanto, além do custo computacional, este processo pode obter redes com conexões desnecessárias.

Outra contribuição deste trabalho, foi o uso de um método de redução de dados, aplicado no estudo de padrões climáticos mensais e sazonais, usando a teoria dos conjuntos aproximativos (redução de dados) e redes neurais (previsão) na derivação de modelos de previsão, a fim de realizar a previsão de precipitação, na região Sul do Brasil.

Levando-se em consideração os resultados apresentados nas Subseções 4.1 e 4.2, constata-se que as melhores soluções para o processo de previsão de precipitação, foram as redes neurais treinadas com o conjunto reduzido de variáveis - ver Figuras 2(c) e 3(c). 


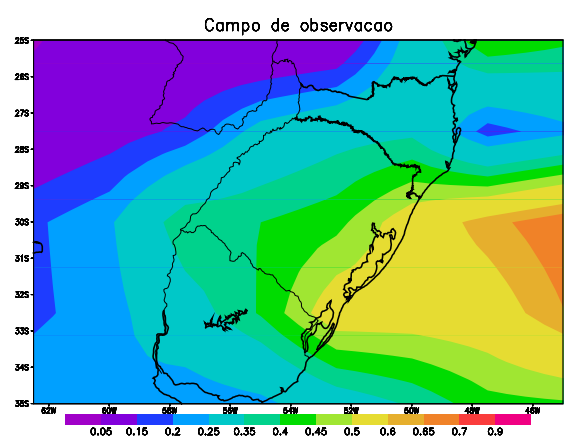

(a) Campo de observação: Inverno

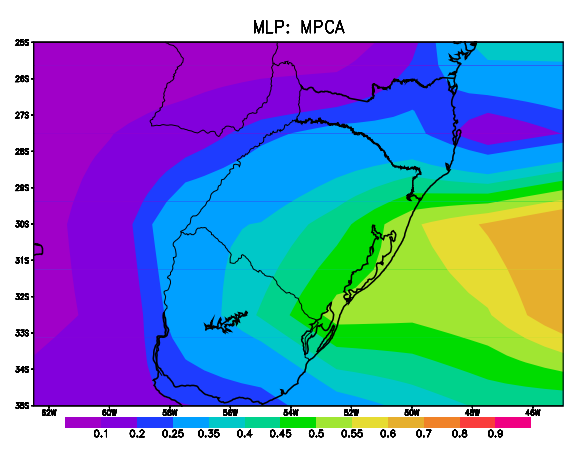

(b) Previsão: NN com todas variáveis

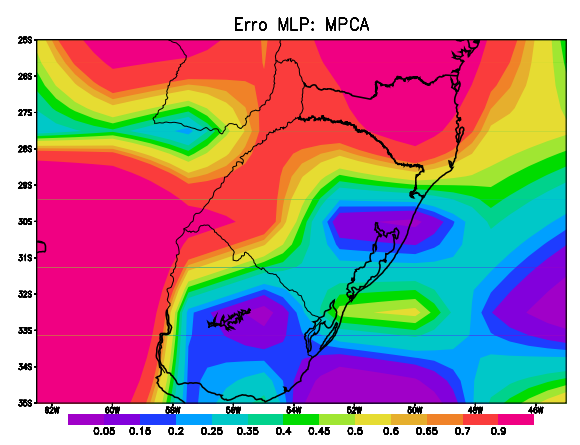

(d) Erro: NN

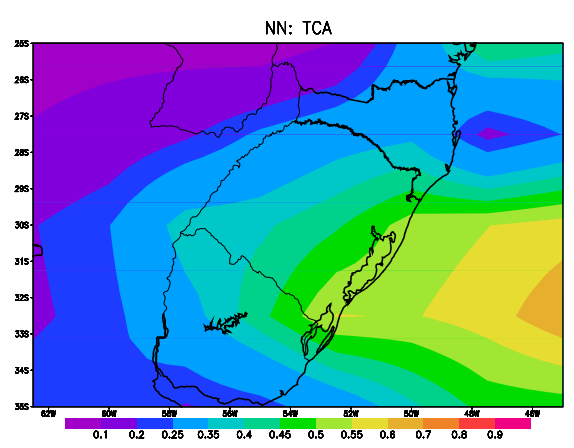

(c) Previsão: NN com redução TCA

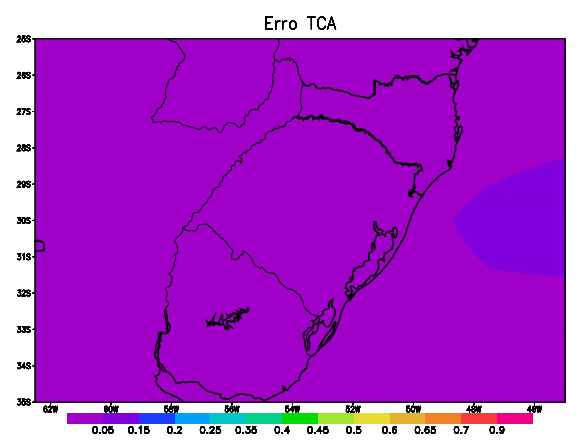

(e) erro: NN TCA

Figura 3: Previsão sazonal de precipitação para estação Inverno

Assim, a técnica TCA aplicada no processo de redução de dimensionalidade de dados, permite a identificação de informações relevantes presentes nos dados para a previsão do climática. Além disso, é uma técnica que aborda o problema de lidar com grandes quantidades de dados, que é uma característica dos processos em meteorologia.

\section{Agradecimentos}

Os autores agradecem ao CNPq e à CAPES.

\section{Referências}

Anochi, J. (2010). Modelos baseados em redes neurais para o estudo de padrões climáticos a partir de dados tratados com a teoria dos conjuntos. Dissertação de Mestrado, Instituto Nacional de Pesquisas Espaciais, São José dos Campos.

Anochi, J., Silva, J. (2009). Uso de redes neurais artificiais e teoria de conjuntos aproximativos no estudo de padrões climáticos sazonais. Learning and Nonlinear Models, 7, 83-91.

Baboo, S. S., Shereef, I. K. (2010). An efficient weather forecasting system using artificial neural network. International journal of environmental science and development, $1(4), 321-326$. 
Carvalho, A. R., Ramos, F. M., Chaves, A. A. (2011). Metaheuristics for the feedforward artificial neural network architecture optimization problem. Neural Computing and Applications, 20(8), 1273-1284.

Haykin, S. (1998). Neural networks principles and practices, $2^{\circ}$ edn. Prentice Hall, Canada.

Komorowski, J., Pawlak, Z., Polkowski, L., Skowron, A. (1998). Rough Set: a tutorial. Department of Computer and Information Science Norwegian University os Science and Technology (NTNU), Polond.

Luz, E., Becceneri, J., Velho, H. (2008). A new multiparticle collision algorithm for otimization in a highperformance environment. Journal of Computacional Interdisciplinary Sciences, 1, 1-7.

Øhrn, A., Komorowski, J. (1997). Rosetta - a rough set toolkit for analysis of data. Em: Proceedings..., International Joint Conference on Information Sciences, Institute of Computer Science, Durham, USA, pp. 403407.

Pawlak, Z. (1982). Rough sets. International Journal of Computer $\mathcal{E}$ Information Sciences, 11(5), 341-356.

Prechelt, L. (1994). ROSETTA technical reference manual. Proben1: A set of neural network benchmark problems and benchmarking rules, Germany.

Sampaio, G., Silva Dias, P. L. (2014). Evolução dos modelos climáticos e de previsão de tempo e clima. Revista USP, 2(103), 41-54.

Sousa, W., Sousa, F. (2010). Rede neural artificial aplicada à previsão de vazão da bacia hidrográfica do rio piancó. Revista Brasileira de Engenharia Agrícola e Ambiental, 14(2), 173-180.

Autores: Previsão climática por rede neural 